\title{
Combined inhibition of EMMPRIN and epidermal growth factor receptor prevents the growth and migration of head and neck squamous cell carcinoma cells
}

\author{
SHINSUKE SUZUKI and KAZUO ISHIKAWA \\ Department of Otorhinolaryngology and Head and Neck Surgery, \\ Akita University Graduate School of Medicine, Akita 010-8543, Japan \\ Received November 11, 2013; Accepted December 9, 2013
}

DOI: $10.3892 /$ ijo.2013.2238

\begin{abstract}
It has been reported that the epidermal growth factor receptor (EGFR) expression is associated with the extracellular matrix metalloproteinase inducer (EMMPRIN) in some solid tumors; however, the relationship of EMMPRIN with EGFR in head and neck cancers is not fully understood. To determine the relationship between EMMPRIN and EGFR in head and neck squamous cell carcinoma (HNSCC), HNSCC cells were stimulated with epidermal growth factor (EGF), a ligand of EGFR. EMMPRIN expression in HNSCC cells was upregulated by EGF. In addition, EGF stimulation induced HNSCC cell invasion and MMP-9 expression. This increase in invasion and MMP-9 expression was abrogated by downmodulation of EMMPRIN. Furthermore, to determine the effects of combined EMMPRIN and EGFR targeting in HNSCC, HNSCC cells were treated with an EMMPRIN function-blocking antibody and the EGFR inhibitor AG1478. This combined treatment resulted in greater inhibition of HNSCC cell proliferation and migration compared with the individual agents alone. These results suggest that EMMPRIN mediates EGFR-induced tumorigenicity and that combined targeting of EMMPRIN and EGFR may be an efficacious treatment approach.
\end{abstract}

\section{Introduction}

Head and neck squamous cell carcinoma (HNSCC) is the most common malignancy of the upper aerodigestive tract that is characterized by a propensity for invasion and cervival lymph node metastasis. In spite of advances in surgery, radiation, and chemotherapy, HNSCCs continue to have poor outcomes

Correspondence to: Dr Shinsuke Suzuki, Department of Otorhinolaryngology and Head and Neck Surgery, Akita University Graduate School of Medicine, Akita 010-8543, Japan

E-mail: suzukis@med.akita-u.ac.jp

Key words: extracellular matrix metalloproteinase inducer, epidermal growth factor receptor, head and neck cancer, matrix metalloproteinase, invasion, migration, proliferation and are still often fatal. Therefore, new and more efficacious therapies are needed to improve HNSCC survival (1).

The epidermal growth factor receptor (EGFR) is a receptor tyrosine kinase that regulates crucial cellular signaling pathways contributing to tumor progression $(2,3)$ and is frequently amplified and overexpressed in a high percentage of HNSCCs $(4,5)$. Preclinical studies have successfully demonstrated the antitumor effects of EGFR targeting, and the Food and Drug Administration (FDA) has approved the clinical use of EGFR monoclonal antibody cetuximab in HNSCC (6). However, when used alone, EGFR targeting shows insufficient suppression of HNSCCs $(7,8)$; hence, various combination strategies are under investigation with an aim to improve HNSCC treatments and prognosis (9-11).

Accumulating studies have showed that EGFR stimulation induces matrix metalloproteinase (MMP) expression, which degrade extracellular matrix and is critical for tumor development $(12,13)$. Recently, extracellular matrix metalloproteinase inducer (EMMPRIN), also known as CD147, has been identified as a member of the immunoglobulin superfamily. In particular, EMMPRIN has been reported to be highly expressed in malignant tumors (14-16), including HNSCCs (17) and has been found to induce tumor progression through MMP expression (18-20). Although it has been reported that EGFR expression is associated with EMMPRIN in some solid tumors $(21,22)$, the relationship between EMMPRIN and EGFR in oncogenicity is not fully understood, particularly in head and neck cancers.

This study was undertaken to evaluate the relationship between EMMPRIN and EGFR and to test the hypothesis that EMMPRIN mediates EGFR-induced HNSCC tumorigenic behavior to determine if combined inhibition of EMMPRIN and EGFR is a rational treatment approach to improve prognosis in HNSCC.

\section{Materials and methods}

Cell and cell culture. The HNSCC cell line SAS, a human tongue squamous cell carcinoma cell line, was used. Cells were maintained in Dulbecco's modified Eagle's medium (DMEM) with $10 \%$ heat-inactivated fetal bovine serum (FBS) and incubated at $37^{\circ} \mathrm{C}$ in the presence of $5 \% \mathrm{CO}_{2}$. 

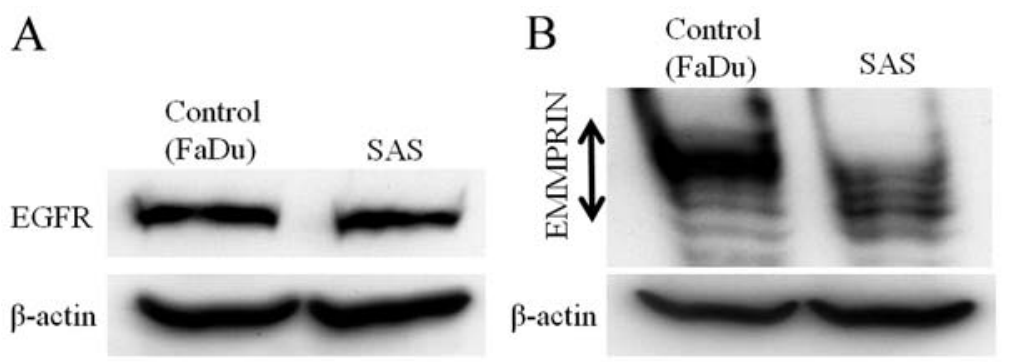

Figure 1. The HNSCC cell line SAS expresses EGFR and EMMPRIN. EGFR and EMMPRIN protein expression was detected by immunoblotting in the HNSCC SAS cell line. EGFR, epidermal growth factor receptor; EMMPRIN, extracellular matrix metalloproteinase inducer; HNSCC, head and neck squamous cell carcinoma.

Western blotting. Protein expression was detected by western blotting. Cells were lysed in detergent, and protein levels were determined using the Bio-Rad protein assay method. Total protein was separated on $8 \%$ sodium dodecyl sulfatepolyacrylamide gel electrophoresis (SDS-PAGE) gel and transferred to a nitrocellulose membrane. The blot was incubated with each antibody and developed using the Luminor Regent (Santa Cruz Biotechnology).

Gelatin zymography. Gelatinase expression was detected in conditioned media by gelatin zymography. Cells were cultured for $48 \mathrm{~h}$ in serum-free DMEM with or without epidermal growth factor (EGF). This was followed by the detection of gelatinolytic activity in the conditioned media by gelatin zymography. Using a $7.5 \%$ separating gel containing $0.3 \mathrm{mg} / \mathrm{ml}$ gelatin, the conditioned medium was resolved by SDS-PAGE under non-reducing conditions. The gels were washed and then incubated for $24 \mathrm{~h}$ at $37^{\circ} \mathrm{C}$ in a reaction buffer before being stained. After destaining, gelatinolytic activity on the gel was detected as clear bands on a blue background of undigested gelatin.

Small interfering RNA (siRNA) and siRNA transfection. EGFR siGENOME SiRNA, EMMPRIN siGENOME SiRNA (Dharmacon RNA Technologies, Lafayette, CO, USA) was transfected into HNSCC cells for each protein silencing. The siGENOME non-targeting siRNA was used as a control. The siRNA transfections were performed using Lipofectamine 2000 (Life Technology Inc.).

Matrigel invasion and cell migration assays. Cell invasion was evaluated in vitro using Matrigel-coated semipermeable modified Boyden inserts with a pore size of $8 \mu \mathrm{m}$ (BectonDickinson/Biocoat, Bedford, MA, USA). Cell migration was evaluated in vitro using semipermeable modified Boyden inserts with a pore size of $8 \mu \mathrm{m}$ (Becton-Dickinson/Biocoat). For each assay, cells were plated in duplicate at a density of $5 \times 10^{3}$ cells/well for the invasion assay or $3 \times 10^{4}$ cells/well for the migration assay. Plating was carried out on serum-free DMEM with $100 \mathrm{nM}$ EGF for the invasion assay, or either the control vehicle (DMSO), AG1478 $(10 \mu \mathrm{M})$, anti-EMMPRIN function-blocking antibody $(10 \mu \mathrm{g} / \mathrm{ml})$, or a combination of AG1478 and anti-EMMPRIN for the migration assay in the inserts. The cells were plated in 96-well plates to serve as loading controls. Both the insert and the holding well were subjected to the same medium composition with the exception of serum. The insert contained no serum, whereas the lower well contained $10 \%$ FBS that served as a chemoattractant. After $24-\mathrm{h}$ treatment at $37^{\circ} \mathrm{C}$ in a $5 \% \mathrm{CO}_{2}$ incubator, the cells in the insert were removed by gentle wiping using a cotton swab. Cells on the reverse side of the insert were fixed and stained with Diff-Quik ${ }^{\circledR}$ (Sysmex, Kobe, Japan) according to the manufacturer's instructions. Cells plated in 24-well plates were subjected to 3-(4,5-dimethylthiazol-2-yl)-2,5-diphenyltetrazolium bromide assays, and the cell number across the groups were normalized. The number of invading or migrating cells was adjusted accordingly.

Proliferation assay. SAS cells were plated in triplicate at a density of $3 \times 10^{4}$ cells/well and allowed to seed overnight in a 12-well plate. Cells were then treated with either the control vehicle (DMSO), AG1478 $(10 \mu \mathrm{M})$, anti-EMMPRIN functionblocking antibody $(10 \mu \mathrm{g} / \mathrm{ml})$, or a combination of AG1478 and anti-EMMPRIN antibody in DMEM with $10 \%$ FBS. At selected time-points, cells were trypsinized and stained with trypan blue, and viable cells were counted using a hemocytometer.

Statistical analysis. The statistical significance of differences was assessed using Wilcoxon-Mann-Whitney 2-tailed exact test.

\section{Results}

HNSCC cell line SAS expresses EGFR and EMMPRIN. To evaluate the role of the interaction between EGFR and EMMRPIN, both protein expressions were determined by immunoblotting in the HNSCC cell line SAS. FaDu cells, in which the expression of EGFR and EMMPRIN has been reported, were used as the control $(23,24)$ (Fig. 1).

EGF induces EMMPRIN and MMP-9 expression. Coexpression of EGFR and EMMPRIN in clinical samples has been identified for its importance for tumor progression or prognosis in several solid tumors $(21,22)$; however, the relationship between these factors have not been studied completely. Therefore, EGF, a ligand of EGFR, was added to the culture media at various concentrations to stimulate EGFR. EMMPRIN expression was determined by immunoblotting and the expression of gelatinase, MMP-2 and MMP-9, which plays an important role in tumor invasion and metastasis through basement membrane was detected by 


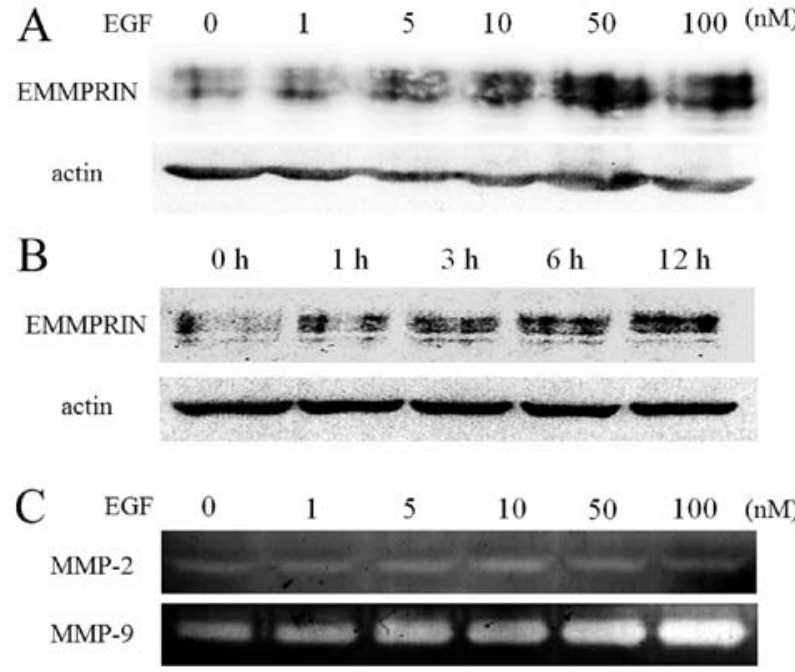

Figure 2. EGF increases EMMPRIN and MMP-9 expression. SAS cells were plated and allowed to seed overnight in a 6-well plate. The cells were grown in serum-free DMEM for $24 \mathrm{~h}$ prior to EGF treatment. Next, the cells were treated with serum-free DMEM containing increasing concentrations of EGF (0-100 nM) for $24 \mathrm{~h}$ or $10 \mathrm{nM}$ for $0-12 \mathrm{~h}$. The cells were lysed and subjected to immunoblotting to determine EMMPRIN protein expression, EGF increased EMMPRIN expression dose- (A) and a time-dependently (B). SAS cells were also treated with $10 \mathrm{nM}$ for $24 \mathrm{~h}$. The culture medium was harvested followed by gelatin zymography for the evaluation of gelatinase activity, EGF induced MMP-9 expression, but not MMP-2 expression, in the culture media in a dose-dependent manner (C). DMEM, Dulbecco's modified Eagle's medium; EGF, epidermal growth factor; EGFR, epidermal growth factor receptor; EMMPRIN, extracellular matrix metalloproteinase inducer; HNSCC, head and neck squamous cell carcinoma.
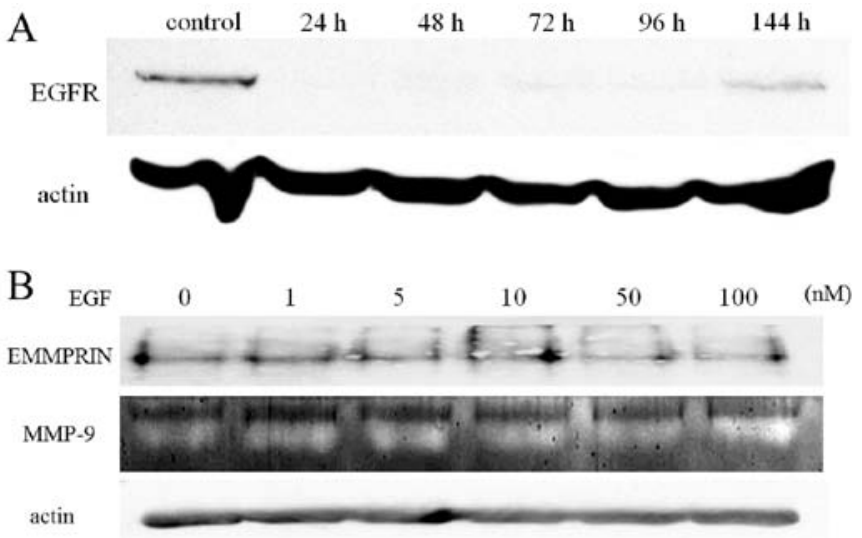

Figure 3. EGFR mediates EGF-induced EMMPRIN and MMP-9 upregulation. SAS cells were transfected with EGFR siRNA, EGFR expression was abolished from $24 \mathrm{~h}$ until $144 \mathrm{~h}$ after siRNA transfection (A). Twentyfour hours after EGFR siRNA transfection, increasing concentration of EGF was added to the media as in Fig. 2. The upregulation of EMMPRIN and MMP-9, shown in Fig. 2, was not observed during the 24-h incubation (B). EGF, epidermal growth factor; EGFR, epidermal growth factor receptor; EMMPRIN, extracellular matrix metalloproteinase inducer; MMP-9, matrix metallopeptidase 9; siRNA, small interfering RNA.

gelatin zymography. EGF increased EMMPRIN expression in a dose- (Fig. 2A) and time- (Fig. 2B) dependent manner. Interestingly, EGF induced MMP-9 expression, and not MMP-2 expression, in culture media in a dose-dependent manner (Fig. 2C).
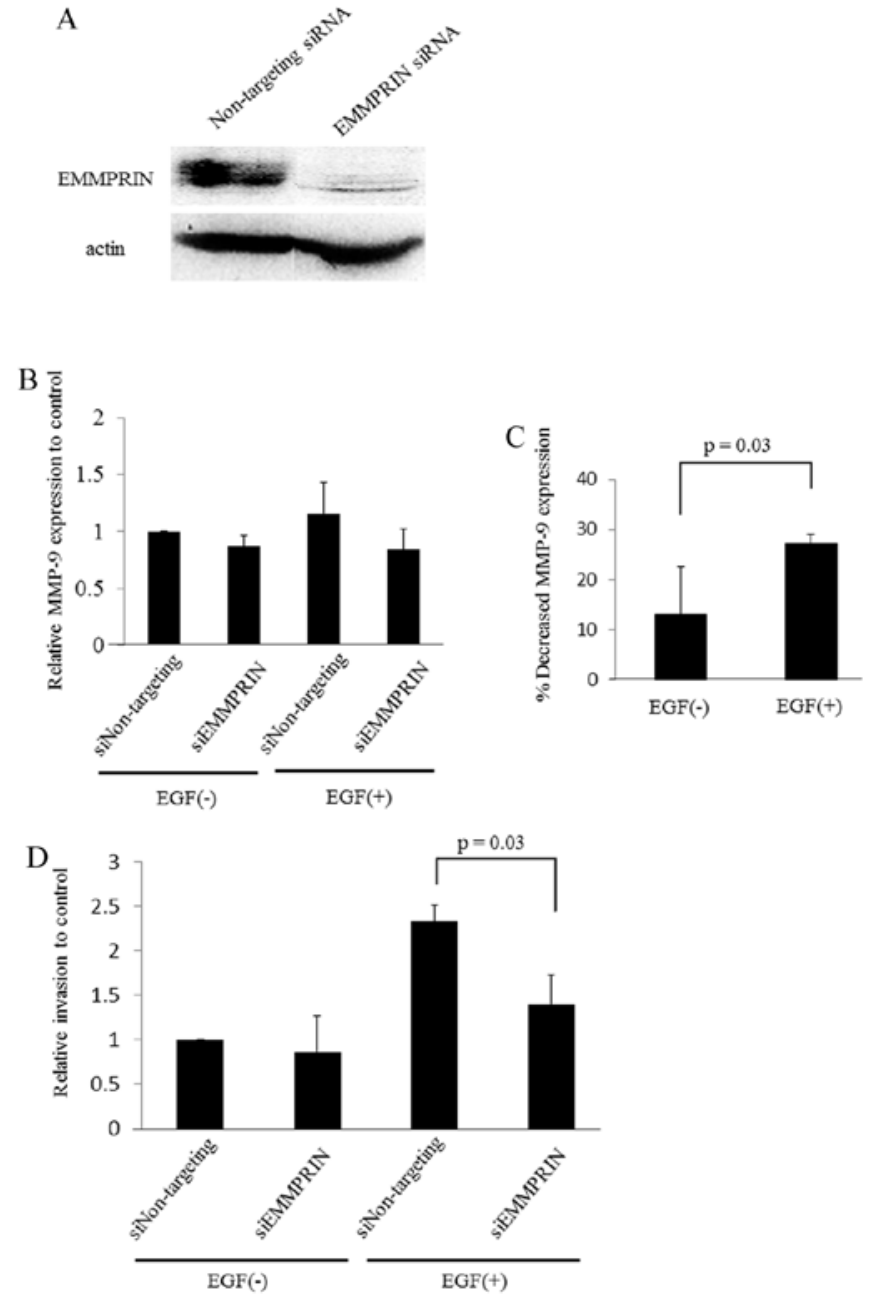

Figure 4. EMMPRIN plays a critical role in EGFR-induced MMP-9 expression and invasion. SAS cells were transfected with non-targeting siRNA or EMMPRIN siRNA and cultured with or without $100 \mathrm{nM}$ EGF (A). The MMP-9 expression in HNSCC cells decreased when cells were treated with EMMPRIN siRNA compared with non-targeting siRNA either in the presence or absence of EGF (B). Percent reduction in MMP-9 with EMMPRIN siRNA was calculated from the results shown in (B). The degree of MMP-9 reduction with EMMPRIN siRNA was greater under EGF-treated conditions (C). Cells were also subjected to an invasion assay. Cells were plated on inserts with or without $100 \mathrm{nM}$ EGF in serum-free media with the lower well containing 10\% FBS serving as a chemoattractant, EMMPRIN siRNA decreased HNSCC invasion during EGF treatment (D). EGF, epidermal growth factor; EGFR, epidermal growth factor receptor; EMMPRIN, extracellular matrix metalloproteinase inducer; HNSCC, head and neck squamous cell carcinoma; MMP-9, matrix metallopeptidase 9; siRNA, small interfering RNA.

EGFR mediates EGF-induced EMMPRIN and MMP-9 upregulation. The results shown in Fig. 2 indicate that EGF induces EMMPRIN and MMP-9 expression in the HNSCC cell line. Although EGF is a representative ligand for EGFR, it is unclear whether EGF induces the phenomena mentioned in Fig. 2. To confirm whether EGFR mediates EGF-induced EMMPRIN and MMP-9 upregulation in the cells, we used siRNA on EGFR before culturing cells in the presence or absence of EGF. As shown in Fig. 3B, the upregulation of EMMPRIN and MMP-9 induced by EGF, shown in Fig. 2A and B, were not observed when EGFR was suppressed by siRNA. This result indicates 

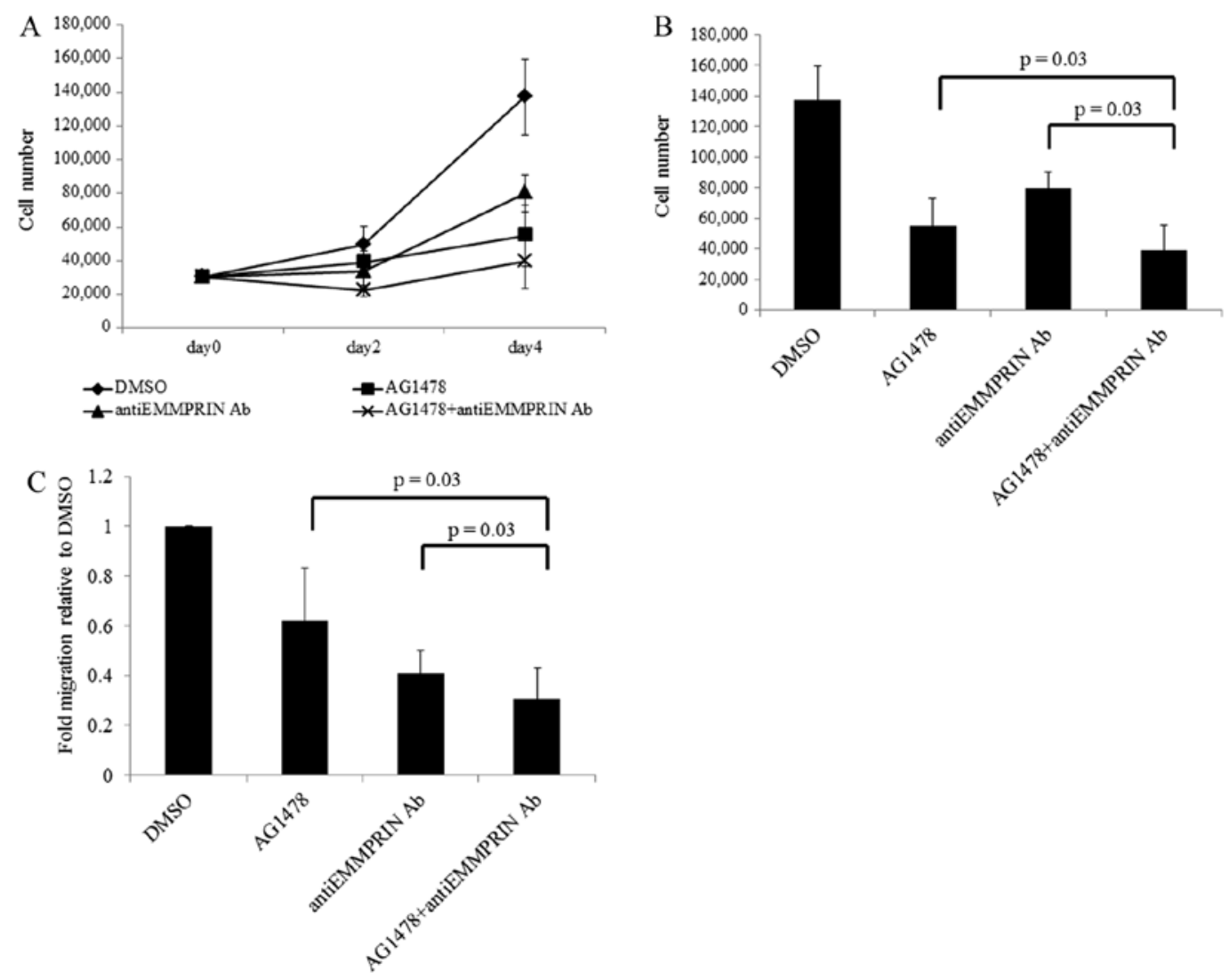

Figure 5. Combined inhibition of EMMPRIN and EGFR inhibits growth and migration of SAS cells. (A) A proliferation assay of SAS HNSCC cells treated with AG1478 and/or anti-EMMPRIN function-blocking antibody. After $24 \mathrm{~h}$, cells were plated in 12-well plates in DMEM with 10\% FBS, and the growth media was replaced with media containing each agent. Cells were harvested and counted by vital dye exclusion. Cell counts on days 2 and 4 from 3 independent experiments are presented. (B) Cell numbers on day 4 in each condition are presented. (C) For migration experiments, SAS cells were plated in inserts and cultured with each agent in DMEM with 10\% FBS, After 48-h incubation, migrating cells on the reverse side of the insert were counted using light microscopy. The experiment was repeated 3 times with similar results. Proliferation and invasion of SAS cells treated with the combination of AG1478 and anti-EMMPRIN antibody decreased to $29.0 \%( \pm 12.6 \%)$ and $30.6 \%( \pm 12.5 \%)$, respectively, of the control vehicle. This was lower than AG1478 alone $[41.1 \pm 17.7 \%(\mathrm{P}=0.03)$ and $61.9 \pm 21.3 \%(\mathrm{P}=0.03)$, respectively] or anti-EMMPRIN antibody alone $[60.0 \pm 15.3 \%(\mathrm{P}=0.03)$ and $40,9 \pm 9,1 \%(\mathrm{P}=0.03)$, respectively] using the Wilcoxon-Mann-Whitney 2-tailed exact test. EGF, epidermal growth factor; EGFR, epidermal growth factor receptor; EMMPRIN, extracellular matrix metalloproteinase inducer; FBS, fetal bovine serum; HNSCC, head and neck squamous cell carcinoma; MMP-9, matrix metallopeptidase 9; siRNA, small interfering RNA.

that EGF stimulates EGFR and that this interaction resulted in the upregulation of EMMPRIN and MMP-9.

EMMPRIN plays a critical role in EGFR-induced MMP-9 expression and cell invasion. The ability of EGFR to promote tumor progression is well documented (2,3,25-27). In addition, accumulating studies have reported that EMMPRIN induces malignant phenotypes such as invasion, proliferation, and MMP expression in some solid tumors including HNSCCs (28-31). Based on these reports and our findings shown in Fig. 3, we hypothesized that EGFR stimulation induces EMMPRIN expression, which induces malignant phenotypes in SAS. To investigate the importance of EMMPRIN in the EGFRinduced tumorigenic process, we analyzed MMP-9 expression and cell invasion in the presence or absence of siRNA-targeting EMMPRIN, with or without EGF in the cell culture media.

The results showed a trend for SAS to decrease MMP-9 expression when EMMPRIN was knocked-down in either the presence or absence of EGF (Fig. 4B). However, the percent decrease in MMP-9 was significantly higher in the presence of EGF (Fig. 4C). This suggests that EMMPRIN plays an important role in MMP-9 expression induced by EGFR stimulation and that both EGFR and EMMPRIN are crucial for MMP-9 expression in SAS. In addition, cell invasiveness was significantly decreased when EMMPRIN was silenced by siRNA under EGFR stimulation by EGF (Fig. 4D), indicating that EMMPRIN has a key role in EGFR-induced HNSCC cell invasion.

Combined inhibition of EGFR and EMMPRIN prevents the growth and migration of HNSCC cells. The aforementioned results indicate that EGFR induces HNSCC malignant phenotypes through EMMPRIN. Indeed, it is well known that EGFR induces oncogenicity (32) and that its targeting is effective in managing various solid tumors (33). In addition, both EMMPRIN tumorigenicity and the antitumor effect of EMMPRIN inhibition have been investigated previously $(34,35)$. Hence, we examined the combined effect of EGFR and EMMPRIN in HNSCC cell proliferation and migration.

To inhibit EGFR and EMMPRIN function, cells were treated with or without the EGFR antibody AG1478 and/or the EMMPRIN function-blocking antibody. As shown in Fig. 5B 
and $\mathrm{C}$, cell proliferation and migration were significantly decreased by both AG1478 and EMMPRIN function-blocking antibody. Furthermore, combined inhibition showed a more marked and significant reduction in proliferation and migration when compared with single inhibition of EGFR and EMMPRIN.

\section{Discussion}

This study demonstrated that in HNSCC, EMMPRIN partly promotes increased MMP-9 expression and cell invasion induced by EGF stimulation through EGFR. Furthermore, combined inhibition of EMMPRIN and EGFR shows effective suppression of HNSCC proliferation and migration. These results suggest the need for further investigation into the mechanism of combined EMMPRIN and EGFR targeting in cancers that express increased levels of these proteins.

HNSCC is the most common malignancy of the upper aerodigestive tract (36), and despite advancements in conventional treatment, the prognosis has remained unchanged over the past several decades with $30 \%$ patient deaths every year (37). Therefore, new and more effective therapeutic approaches are needed to improve HNSCC survival (1). Recently, molecular targeting has been introduced for the treatment of malignancy, including HNSCC (38). EGFR is one such target that is thought to be an ideal therapeutic target (39) because it is frequently amplified and overexpressed in high percentage of HNSCCs (4). However, the use of EGFR inhibitors as monotherapy have yielded low response rates in clinical practice (8). Although it has been reported that an EGFR variant is a possible reason for resistance to EGFR targeting in HNSCCs $(37,40)$, the tumor features that contribute to EGFR targeting resistance are incompletely understood. A promising solution to improve the clinical response rate may be through the combination of EGFR inhibitors with other treatment modalities. Some reports have showed promising antitumor efficacy by blocking EGFR with other tumorigenic factors, such as c-Met (11), Src (10), signal transducer and activator of transcription 3 (STAT3) (9) and G-protein-coupled receptor (GPCR) (41).

The importance of EMMPRIN in tumor progression and its association with poor prognosis is widely known in solid tumors including HNSCC. In addition, there are accumulating reports suggesting that EMMPRIN is a negative prognostic factor in malignant tumors (42-44). We previously reported that the EMMPRIN-EMMPRIN hemophilic interaction or EMMPRIN-cyclophilin A interaction play an important role in MMP expression and activation, as well as in invasion and migration $(24,28)$. Recently, several studies have showed that EMMPRIN blocking or silencing is efficient for cancer suppression $(35,45)$, particularly in HNSCCs, where there have been several reports showing the possibility of EMMPRIN targeting therapy for tumor suppression in vivo. Hence, it appears that there is a more detailed mechanism underlying EMMPRIN-mediated cancer progression and the relationship with other oncogenic factors that contribute to an improvement in HNSCC progression.

The relationship between EMMPRIN, MMP, and EGFR have been reported in colon (21) and breast (22) cancers. In these studies, increased EGFR expression correlated with MMP-9 and EMMPRIN expression in clinical samples, but details of the interaction mechanisms between EGFR,
MMP-9, and EMMPRIN expression were not described. Here we showed that EGFR induces EMMPRIN expression, with EMMPRIN mediating EGFR-induced MMP-9 expression and HNSCC invasion. Our results may account for the expression mechanisms of EGFR, MMP-9, and EMMPRIN in previous studies. However, EGFR silencing did not abolish the expression of EMMPRIN (data not shown). These results suggest that the expression of EMMPRIN is not completely dependent on EGFR expression and that there may be an independent EMMPRIN tumorigenic pathway involved. Therefore, blocking of both EGFR and EMMPRIN may be necessary to regulate HNSCC progression.

In this study, we showed that the combined inhibition of EGFR and EMMPRIN effectively reduces HNSCC cell proliferation and migration. Therefore, complementary blockade of EGFR and EMMPRIN oncogenic pathways might provide a more efficacious treatment approach. Further research into the role of EMMPRIN, and of the apparent synergistic effect, may contribute to improved prognoses in HNSCC.

\section{Acknowledgements}

This study was supported by JSPS KAKENHI grant no. 24791740.

\section{References}

1. Leon $\mathrm{X}$, Hitt $\mathrm{R}$, Constenla $\mathrm{M}$, et al: A retrospective analysis of the outcome of patients with recurrent and/or metastatic squamous cell carcinoma of the head and neck refractory to a platinum-based chemotherapy. Clin Oncol 17: 418-424, 2005

2. Carpenter G and Cohen S: Epidermal growth factor. J Biol Chem 265: 7709-7712, 1990

3. Mendelsohn $\mathrm{J}$ and Baselga $\mathrm{J}$ : The EGF receptor family as targets for cancer therapy. Oncogene 19: 6550-6565, 2000.

4. Grandis JR and Tweardy DJ: Elevated levels of transforming growth factor alpha and epidermal growth factor receptor messenger RNA are early markers of carcinogenesis in head and neck cancer. Cancer Res 53: 3579-3584, 1993.

5. Santini J, Formento JL, Francoual M, et al: Characterization, quantification, and potential clinical value of the epidermal growth factor receptor in head and neck squamous cell carcinomas. Head Neck 13: 132-139, 1991.

6. Bonner JA, Harari PM, Giralt J, et al: Radiotherapy plus cetuximab for squamous-cell carcinoma of the head and neck. N Engl J Med 354: 567-578, 2006.

7. Cohen EE, Rosen F, Stadler WM, et al: Phase II trial of ZD1839 in recurrent or metastatic squamous cell carcinoma of the head and neck. J Clin Oncol 21: 1980-1987, 2003.

8. Soulieres D, Senzer NN, Vokes EE, Hidalgo M, Agarwala SS and Siu LL: Multicenter phase II study of erlotinib, an oral epidermal growth factor receptor tyrosine kinase inhibitor, in patients with recurrent or metastatic squamous cell cancer of the head and neck. J Clin Oncol 22: 77-85, 2004.

9. Sen M, Joyce S, Panahandeh M, et al: Targeting Stat3 abrogates EGFR inhibitor resistance in cancer. Clin Cancer Res 18: 4986-4996, 2012.

10. Koppikar P, Choi SH, Egloff AM, et al: Combined inhibition of c-Src and epidermal growth factor receptor abrogates growth and invasion of head and neck squamous cell carcinoma. Clin Cancer Res 14: 4284-4291, 2008.

11. Xu H, Stabile LP, Gubish CT, Gooding WE, Grandis JR and Siegfried JM: Dual blockade of EGFR and c-Met abrogates redundant signaling and proliferation in head and neck carcinoma cells. Clin Cancer Res 17: 4425-4438, 2011.

12. Barsky SH, Siegal GP, Jannotta F and Liotta LA: Loss of basement membrane components by invasive tumors but not by their benign counterparts: Lab Invest 49: 140-147, 1983.

13. Liotta LA, Tryggvason K, Garbisa S, Hart I, Foltz CM and Shafie S: Metastatic potential correlates with enzymatic degradation of basement membrane collagen. Nature 284: 67-68, 1980. 
14. Caudroy S, Polette M, Tournier JM, et al: Expression of the extracellular matrix metalloproteinase inducer (EMMPRIN) and the matrix metalloproteinase-2 in bronchopulmonary and breast lesions. J Histochem Cytochem 47: 1575-1580, 1999.

15. Polette M, Gilles C, Marchand V, et al: Tumor collagenase stimulatory factor (TCSF) expression and localization in human lung and breast cancers. J Histochem Cytochem 45: 703-709, 1997.

16. Muraoka K, Nabeshima K, Murayama T, Biswas C and Koono M: Enhanced expression of a tumor-cell-derived collagenase-stimulatory factor in urothelial carcinoma: its usefulness as a tumor marker for bladder cancers. Int J Cancer 55: 19-26, 1993.

17. Rosenthal EL, Shreenivas S, Peters GE, Grizzle WE, Desmond R and Gladson CL: Expression of extracellular matrix metalloprotease inducer in laryngeal squamous cell carcinoma, Laryngoscope 113: 1406-1410, 2003.

18. Li R, Huang L, Guo H and Toole BP: Basigin (murine EMMPRIN) stimulates matrix metalloproteinase production by fibroblasts J Cell Physiol 186: 371-379, 2001.

19. Sameshima T, Nabeshima K, Toole BP, et al: Glioma cell extracellular matrix metalloproteinase inducer (EMMPRIN) (CD147) stimulates production of membrane-type matrix metalloproteinases and activated gelatinase A in co-cultures with brain-derived fibroblasts. Cancer Lett 157: 177-184, 2000.

20. Guo H, Zucker S, Gordon MK, Toole BP and Biswas C: Stimulation of matrix metalloproteinase production by recombinant extracellular matrix metalloproteinase inducer from transfected Chinese hamster ovary cells. J Biol Chem 272: 24-27, 1997.

21. Jin JS, Wu CY, Lin YF, et al: Higher expression of epidermal growth factor receptor is associated with extracellular matrix metalloprotease inducer in colorectal adenocarcinoma: tissue microarray analysis of immunostaining score with clinicopathological parameters. Dis Markers 22: 309-316, 2006.

22. Menashi S, Serova M, Ma L, Vignot S, Mourah S and Calvo F: Regulation of extracellular matrix metalloproteinase inducer and matrix metalloproteinase expression by amphiregulin in transformed human breast epithelial cells. Cancer Res 63: 7575-7580, 2003.

23. Toulany M, Dittmann K, Baumann M and Rodemann HP: Radiosensitization of Ras-mutated human tumor cells in vitro by the specific EGF receptor antagonist BIBX1382BS. Radiother Oncol 74: 117-129, 2005.

24. Takahashi M, Suzuki S and Ishikawa K: Cyclophilin A-EMMPRIN interaction induces invasion of head and neck squamous cell carcinoma. Oncol Rep 27: 198-203, 2012.

25. Rubin Grandis J, Melhem MF, Gooding WE, et al: Levels of TGF-alpha and EGFR protein in head and neck squamous cell carcinoma and patient survival. J Natl Cancer Inst 90: 824-832, 1998.

26. Ang KK, Berkey BA, Tu X, et al: Impact of epidermal growth factor receptor expression on survival and pattern of relapse in patients with advanced head and neck carcinoma. Cancer Res 62 7350-7356, 2004.

27. Gupta AK, McKenna WG, Weber CN, et al: Local recurrence in head and neck cancer: relationship to radiation resistance and signal transduction. Clin Cancer Res 8: 885-892, 2002.

28. Suzuki S, Sato M, Senoo H and Ishikawa K: Direct cell-cell interaction enhances pro-MMP-2 production and activation in co-culture of laryngeal cancer cells and fibroblasts: involvement of EMMPRIN and MT1-MMP. Exp Cell Res 293: 259-266, 2004
29. Hanata K, Yamaguchi N, Yoshikawa K, et al: Soluble EMMPRIN (extra-cellular matrix metalloproteinase inducer) stimulates the migration of HEp-2 human laryngeal carcinoma cells, accompanied by increased MMP-2 production in fibroblasts. Arch Histol Cytol 70: 267-277, 2007.

30. Bordador LC, Li X, Toole B, et al: Expression of emmprin by oral squamous cell carcinoma. Int J Cancer 85: 347-352, 2000.

31. Sweeny L, Liu Z, Bush BD, Hartman Y, Zhou T and Rosenthal EL: CD147 and AGR2 expression promote cellular proliferation and metastasis of head and neck squamous cell carcinoma. Exp Cell Res 318: 1788-1798, 2012.

32. Nicholson RI, Gee JM and Harper ME: EGFR and cancer prognosis. Eur J Cancer 37 (Suppl 4): S9-S15, 2001.

33. Laskin JJ and Sandler AB: Epidermal growth factor receptor: a promising target in solid tumours. Cancer Treat Rev 30: 1-17, 2004.

34. Tang X, Guo N, Xu L, Gou X and Mi M: CD147/EMMPRIN: an effective therapeutic target for hepatocellular carcinoma. J Drug Target 21: 224-231, 2012.

35. Yang X, Zhang P, Ma Q, et al: EMMPRIN silencing inhibits proliferation and perineural invasion of human salivary adenoid cystic carcinoma cells in vitro and in vivo. Cancer Biol Ther 13: 85-91, 2012.

36. Jemal A, Siegel R, Xu J and Ward E: Cancer statistics, 2010. CA Cancer J Clin 60: 277-300, 2010

37. Sok JC, Coppelli FM, Thomas SM, et al: Mutant epidermal growth factor receptor (EGFRvIII) contributes to head and neck cancer growth and resistance to EGFR targeting. Clin Cancer Res 12: 5064-5073, 2006

38. Goerner M, Seiwert TY and Sudhoff H: Molecular targeted therapies in head and neck cancer - an update of recent developments. Head Neck Oncol 2: 8, 2010.

39. Cassell A and Grandis JR: Investigational EGFR-targeted therapy in head and neck squamous cell carcinoma. Expert Opin Investig Drugs 19: 709-272, 2010.

40. Wheeler SE, Suzuki S, Thomas SM, et al: Epidermal growth factor receptor variant III mediates head and neck cancer cell invasion via STAT3 activation. Oncogene 29: 5135-5145, 2010.

41. Bhola NE, Thomas SM, Freilino M, et al: Targeting GPCRmediated p70S6K activity may improve head and neck cancer response to cetuximab. Clin Cancer Res 17: 4996-5004, 2011.

42. Tian L, Zhang Y, Chen Y, Cai M, Dong H and Xiong L: EMMPRIN is an independent negative prognostic factor for patients with astrocytic glioma. PLoS One 8: e58069, 2013.

43. Zheng HC, Takahashi H,Murai Y, et al: Upregulated EMMPRIN/ CD147 might contribute to growth and angiogenesis of gastric carcinoma: a good marker for local invasion and prognosis. Br J Cancer 95: 1371-1378, 2006.

44. Reimers N, Zafrakas K, Assmann V, et al: Expression of extracellular matrix metalloproteases inducer on micrometastatic and primary mammary carcinoma cells. Clin Cancer Res 10: 3422-3428, 2004.

45. Nakamura K, Kodama J, Hongo A and Hiramatsu Y: Role of EMMPRIN in endometrial cancer. BMC Cancer 12: 191, 2012. 